\title{
Effect of testosterone on the Connexin37 of sexual mature mouse cumulus oocyte complex
}

\author{
Yangyang Zhang, Yang Xu*, Yanrong Kuai, Sheng Wang, Qing Xue and Jing Shang
}

\begin{abstract}
Background: Recent researches demonstrate that pre-treatment with androgen could increase retrieved oocytes number and clinical pregnancy rate in poor ovarian response (POR) patients. In view of gap junction intercellular communication (GJIC) is important for follicular growth, and androgen plays an important role in improving prognosis of POR patients, we speculate that androgen can increase the expression of connexin in follicle cells, and improve ovarian microenvironment, thus can promote ovarian response. The objective of the research is to study the effect of testosterone on connexin37 (CX37) expression so as to provide theoretical basis for adding testosterone in treatment of POR.

Methods: Cumulus-oocyte-cells (COCs) were collected from ICR mice ovaries, and were cultured in vitro for $48 \mathrm{~h}$ and then treated with testosterone (T) at various concentration. To assess whether the effect of androgen on Cx37 expression is mediated through androgen receptor (AR) pathway, COCs were cultured in vitro with Flutamide (androgen receptor antagonist). The expression of $\mathrm{C} \times 37$ was determined by western blot.

Results: The expression of $\mathrm{C} \times 37$ in COCs which were treated with testosterone was higher than that of control group. There were significant differences $(P<0.001 ;<0.001 ;<0.001 ;<0.001)$. Cx37 increased with the elevated testosterone concentrations. Cx37 was lower in androgen receptor antagonist group ( $2.57 \pm 0.12)$ than the corresponding testosterone concentrations group $(4.42 \pm 0.28)$. There were significant differences between two groups $(P<0.001)$.

Conclusions: There was close relationship between gap junction protein and ovarian response, which suggested that androgen could promote ovarian response by increasing the expression of Cx37 in follicle. Androgen plays an important role in ovarian response through the AR pathway and non-AR pathway.
\end{abstract}

Keywords: Androgen, Androgen receptor antagonist, Connexin37, Poor ovarian response

\section{Background}

Poor ovarian response (POR), which is insensitive to ovarian stimulation and results in fewer retrieved oocytes, poorer quality embryos, and lower implantation rate and clinical pregnancy rate, is considered to be one of the most challenging tasks for clinicians in reproductive medicine [1]. POR is common in patients with diminished ovarian reserve (DOR), while the latter is closely related to age. Androgen concentration in ovary decreases sharply as age advances, so we speculate that

\footnotetext{
* Correspondence: xuyangm@126.com

Department of Obstetrics \& Gynecology, Peking University First Hospital, Beijing 100034, China
}

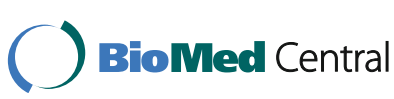

(c) The Author(s). 2016 Open Access This article is distributed under the terms of the Creative Commons Attribution 4.0 International License (http://creativecommons.org/licenses/by/4.0/), which permits unrestricted use, distribution, and reproduction in any medium, provided you give appropriate credit to the original author(s) and the source, provide a link to the Creative Commons license, and indicate if changes were made. The Creative Commons Public Domain Dedication waiver (http://creativecommons.org/publicdomain/zero/1.0/) applies to the data made available in this article, unless otherwise stated. androgen concentration in follicular fluid of POR $\mathrm{pa}-$ tients is lower than normal level. In view of the importance of androgen to follicle recruitment, growth and development, domestic and foreign reproductive centers try to add androgen as an adjuvant to in-vitro fertilization (IVF) treatment protocols in POR patients, in order to increase both quantity and quality of oocytes and embryos, and improve pregnancy outcomes. These interventions include: (1) pretreatment with transdermal testosterone; (2) pretreatment with dehydroepiandrosterone (DHEA); (3) addition of aromatase inhibitors; (4) addition of recombinant luteinizing hormone (LH); (5) addition of human chorionic gonadotrophin (hCG) during 
ovarian stimulation [2]. Commonly used androgen preparations include transdermal testosterone or oral DHEA. The mechanism of action of androgen in improving ovarian response is unclear. Recent researches report that there is a direct and indirect effect of androgen on follicle development. According to the two cell/two gonadotrophin theory, androgen plays an important role in ensuring adequate follicular steroidogenesis. Produced primarily by the theca cells, it acts as a substrate for the aromatase activity of the granlosa cells, and converts to estrogen (estradiol and estrone), sequentially plays a role in follicle growth [3]. This is called indirect regulation effect of androgen. Recent researches report that androgen can also regulate follicle development directly through various mechanisms [4].

Numerous studies have shown that oocyte is in avascular environment, so normal folliculogenesis relies on the bidirectional talk between any two of granulosa cells, cumulus cells and oocytes [5]. As a form of direct pathway, GJIC plays an important role in folliculogenesis and steroidogenesis [6]. Gap junctions are clusters of intercellular channels composed of two compatible hemichannels (connexin, Cx) in the adjacent cells plasma membrane, which permit the rapid exchange of inorganic ions, second messengers and small molecules from cell to cell. Our previous studies have found that a significant decrease of connexin in cumulus cells is closely related to POR. In view of GJIC is vitally important for follicular recruitment, growth and mature, and androgen plays an important role in improving prognosis of POR patients, so we speculate that androgen can increase the expression of connexin in follicle cells, and improve ovarian microenvironment, thus can promote ovarian response.

Current researches about the effect of androgen on gap junction protein mainly focus on polycystic ovary syndrome (PCOS), but the results are not consistent. Cheng et al. found that high androgen reduces connexin43 expression and impairs GJIC between human granulosa cells. High androgen may impair folliculogenesis and in turn lead to ovulatory dysfunction in PCOS patients [7]. But the result of Rabih et al. was in contrast. The study presented that $\mathrm{Cx} 43$ levels were up-regulated in PCO rat ovaries [8]. The researches were aimed at the effect of high concentration of androgen on gap junction protein, and the results still needed further discussion. However, to the best of our knowledge, there has been no report regarding the effect of the different levels with right amount androgen on Cx37 expression in COCs.

The varieties and distributions of connexin in mouse ovary are in accordance with human. Cx43 is mainly localized to the membrane of granulosa cells and cumulus cells, while Cx37 is mainly expressed at the membrane of oocyte in COCs. In this research, we investigated the differences of $\mathrm{Cx} 37$ of COCs which were cultured with different concentrations of androgen among physiological level range, and we explored the mechanism of androgen on ovarian response, and provided theoretical basis for adding androgen in treatment of POR in clinical.

The choice of testosterone concentration in culture medium in vitro is mainly on the basis of physiology concentration of androgen in follicle fluid and cytoxicity test in vitro culture of granulosa cells. Researches suggest that the normal concentration of testosterone in follicle fluid is between $10^{-8} \mathrm{M}$ and $10^{-7} \mathrm{M}$ [9]. Another research about the cytoxicity test in vitro culture of granulosa cells with different concentrations of testosterone suggests that growth inhitibion ratio is about $50 \%$ with $10^{-4} \mathrm{M}$ testosterone. Therefore, the concentration of testosterone in in vitro culture should be lower than $10^{-4} \mathrm{M}$. We treated COCs with testosterone of the equivalent of physiological concentration in follicular fluid $\left(10^{-7} \mathrm{M}\right)$, low concentration $\left(10^{-9} \mathrm{M}, 10^{-11} \mathrm{M}\right)$, high concentration $\left(10^{-5} \mathrm{M}\right)$ after comprehensive evaluation from physiology concentration and cytotoxicology test. To assess whether the effect of androgen on connexin was mediated through the AR pathway, we cultured COCs with $10^{-7} \mathrm{M}$ testosterone and $10^{-6} \mathrm{M}$ Flutamide (androgen receptor antagonist).

\section{Methods}

Twenty six-to-eight-week-old female ICR mice were fed in the barrier environment at animal center of Peking University First Hospital. All animal care requirements were fulfilled, and animals were given food and water ad libitum. After a week, ICR mice were given pregnant mare serum gonadotropin (PMSG, 10 IU per mouse) in order to stimulate ovulation. $48 \mathrm{~h}$ later, ICR mice were put to death through dislocating cervical vertebra. The ovaries were removed and washed in sterile conditions. COCs were isolated from the excised ovaries by follicle puncture using needle in culture medium with dissecting microscope. The culture medium was collected and the cells were sedimented by centrifugation at $500 \mathrm{~g}$ for $5 \mathrm{~min}$ at $4{ }^{\circ} \mathrm{C}$. The COCs were placed in 4-well cell culture plates with $495 \mu \mathrm{L}$ culture medium at a seeding density of 20-30 COCs per well. COCs were cultured in a humidified incubator $\left(5 \% \mathrm{CO}_{2}\right.$ ) at $37{ }^{\circ} \mathrm{C}$ in order to separate from the hormone environment in vivo for $48 \mathrm{~h}$. Then, COCs were treated with $10^{-11} \mathrm{~mol} / \mathrm{L}$ testosterone $(\mathrm{T}$ ) (dissolved by glycerin, $5 \mu \mathrm{L}), 10^{-9} \mathrm{~mol} / \mathrm{L} \mathrm{T}, 10^{-7} \mathrm{~mol} / \mathrm{L} \mathrm{T}$, $10^{-5} \mathrm{~mol} / \mathrm{L} \mathrm{T}, 10^{-0} \mathrm{~mol} / \mathrm{L} \mathrm{T}$ (blank control group), 10 ${ }^{-7} \mathrm{~mol} / \mathrm{L} \mathrm{T}$ and $10^{-6} \mathrm{~mol} / \mathrm{L}$ Flutamide for $24 \mathrm{~h}$. The COCs and culture medium were collected respectively and sedimented by centrifugation at $500 \mathrm{~g}$ for $5 \mathrm{~min}$. The culture medium was discarded and the COCs 
were harvested after being washed with cold phosphate-buffered saline (PBS).

Total proteins extracted from COCs were analyzed by western blot for connexin37. Cells were homogenized into RIPA buffer supplemented with protease inhibitors (100:1) for $30 \mathrm{~min}$, and then centrifuged at $3000 \mathrm{~g}$ for 20 min. The supernatant was transferred to a new centrifuge tube for western blot. After determination of protein content by the bicinchoninic acid (BCA) protein assay method, $30 \mu \mathrm{g}$ proteins were separated by $10 \%$ sodium dodecyl sulfate polyacrylamide gel electrophoresis (SDS-PAGE) and transferred onto nitrocellulose membranes. After blocked with 5\% skimmed milk for $1 \mathrm{~h}$, the membranes were incubated with primary goat antimouse Cx37 antibody (1:200, Santa Cruz, USA) for $1 \mathrm{~h}$ at room temperature and overnight at $4{ }^{\circ} \mathrm{C}$. After washed three times in Tris-buffered saline (TBS)- $0.1 \%$ Tween 20, the membranes were incubated with monkey anti-goat IgG conjugated with horseradish peroxidase (HRP) (1:5000, ZSGB-BIO, China) for $1 \mathrm{~h}$ at room temperature. The membranes were washed again like above and the immunoreactivity was examined by the enhanced chemiluminescence (ECL) system. As a control for normalization, the membranes were re-probed for internal control, $\beta$-actin (1:200, Santa Cruz, USA). Films were scanned, and the optical density of the bands was measured with AlphaEaseFC. The relative quantity of $\mathrm{Cx} 37$ was determined with reference to $\beta$-actin. Final data were expressed as the mean of results in three independent experiments performed at different time points.

All analyses were performed with Software Package for Social Sciences (SPSS) version 10.0 for windows. All data were expressed as mean \pm standard deviation (SD). Comparison between two groups was done with independent sample $T$-test, and comparison among multi samples was done with variance analysis, and intergroup multiple comparison was done with Bonferroni. $P<0.05$ was considered as statistically significant.

\section{Results}

\section{Influence of different concentrations of testosterone on} Cx37 expression

To study the mechanism of androgen improving ovarian response, our research examined the levels of expression of Cx37 in COCs which were cultured with different levels of testosterone for $24 \mathrm{~h}$ in vitro.

Western blot results suggested that Cx37 could be detected in COCs of all groups. The results were shown in Fig. 1. The expression of $\mathrm{Cx} 37$ in COCs which were treated with $10^{-11} \mathrm{~mol} / \mathrm{L} \mathrm{T}(3.55 \pm 0.10), 10^{-9} \mathrm{~mol} / \mathrm{L} \mathrm{T}$ $(3.92 \pm 0.21), 10^{-7} \mathrm{~mol} / \mathrm{L} \mathrm{T}(4.42 \pm 0.28), 10^{-5} \mathrm{~mol} / \mathrm{L} \mathrm{T}$ $(4.93 \pm 0.22)$ was higher than that of blank control group $(1.59 \pm 0.29)$. There were significant differences $(P<$ $0.001 ;<0.001 ;<0.001 ;<0.001)$.

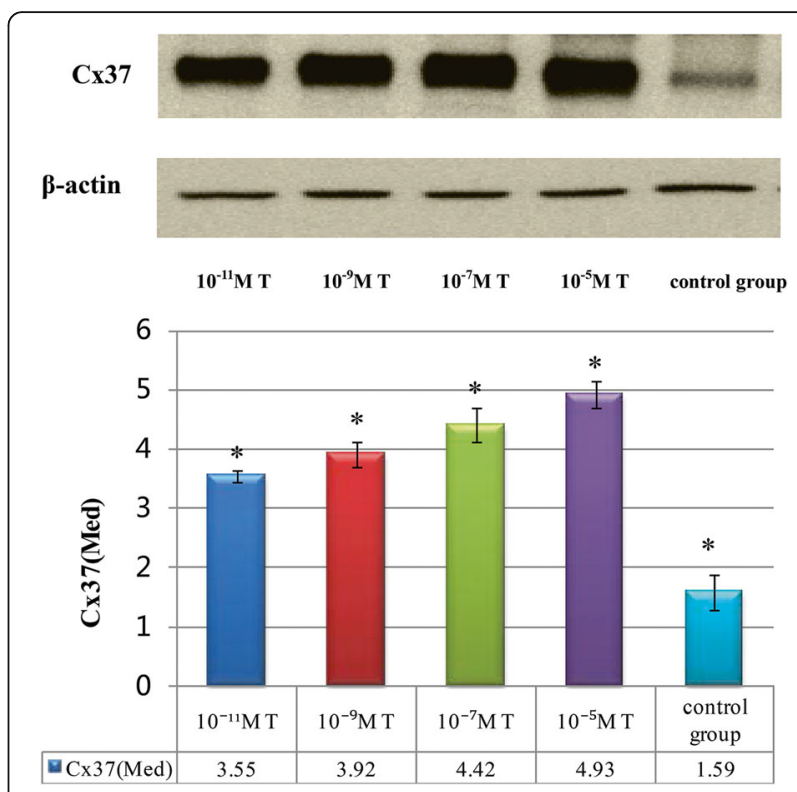

Fig. 1 Expression of $\mathrm{Cx} 37$ in COCs in different concentrations of testosterone. The expression of $\mathrm{C} \times 37$ which were treated with $\mathrm{T}$ was higher than that of blank control group. Cx37 increased with the elevated testosterone concentrations, and there was significantly difference in four groups of being cultured with testosterone

The expression of $\mathrm{Cx} 37$ in COCs increased with the elevated testosterone concentrations, and there was significantly difference in four groups of being cultured with testosterone $(P<0.05)$. Bonferroni analysis suggested that the expression of Cx37 in COCs which was treated with $10^{-11} \mathrm{~mol} / \mathrm{L} \mathrm{T}$ was significantly lower than those of being treated with $10^{-9} \mathrm{~mol} / \mathrm{L} \mathrm{T}$, $10^{-7} \mathrm{~mol} / \mathrm{L} \mathrm{T}, 10^{-5} \mathrm{~mol} / \mathrm{L} \mathrm{T}(P=0.002 ;<0.001 ;<0.001)$. The expression of $\mathrm{Cx} 37$ in COCs which was treated with $10^{-9} \mathrm{~mol} / \mathrm{L} \mathrm{T}$ was significantly lower than those of being treated with $10^{-7} \mathrm{~mol} / \mathrm{L} \mathrm{T}, 10^{-5} \mathrm{~mol} / \mathrm{L} \mathrm{T}(P<$ $0.001 ;<0.001)$. The expression of Cx37 in COCs which was treated with $10^{-7} \mathrm{~mol} / \mathrm{L} \mathrm{T}$ was significantly lower than that of being treated with $10^{-5} \mathrm{~mol} / \mathrm{L} \mathrm{T}(P<0.001)$.

\section{The effect of androgen receptor antagonist (Flutamide) on $\mathrm{C} \times 37$ expression}

To assess whether the effect of androgen on Cx37 expression was mediated through androgen receptor (AR) pathway, COCs were cultured in vitro with testosterone and Flutamide. The physiological concentration of testosterone in follicle fluid is $10^{-7} \mathrm{M}$, so we cultured COCs with $10^{-7} \mathrm{M}$ testosterone and $10^{-6} \mathrm{M}$ Flutamide (10-fold excess above that of testosterone).

Western blot results suggested that Cx37 was detected in COCs in both groups. The expression of Cx37 in COCs which were treated with $10^{-7} \mathrm{~mol} / \mathrm{L} \mathrm{T}$ and $10^{-6} \mathrm{~mol} / \mathrm{L}$ Flutamide $(2.57 \pm 0.12)$ was lower than that of only treated with $10^{-7} \mathrm{~mol} / \mathrm{L} \mathrm{T}(4.42 \pm 0.28)$. 
There were significant differences between two groups $(P<0.001)$. The results were shown in Fig. 2 .

\section{Discussion}

POR is one of the most challenging tasks for clinicians in reproductive medicine. Several interventions including adjusting stimulation protocols, using oral contraceptive, adding growth hormone have been proposed to improve the POR outcome. Unfortunately, the effect of these interventions is limited. Recent researches with encouraging results demonstrate that pre-treatment with androgen, such as DHEA and trans-dermal testosterone, could increase retrieved oocytes number and clinical pregnancy rate in DOR and POR patients. Casson et al. first reported the benefits of DHEA supplementation for improving the ovarian response and retrieved oocytes number [10]. Since then, a few controlled studies including a randomized controlled study, but with small sample size, have subsequently reported benefits of DHEA supplementation to improve ovarian response and IVF outcome [11]. Nevertheless, controversy still exists as to whether these protocols improved cycle outcome $[12,13]$. A recent world-wide survey has shown that over a quarter of reproduction centers add DHEA as an adjuvant to IVF treatment protocols in women with DOR or POR. Despite widespread use of androgen, clinical evidence as well as knowledge regarding underlying mechanisms of androgen on improvement of ovarian response is still limited.

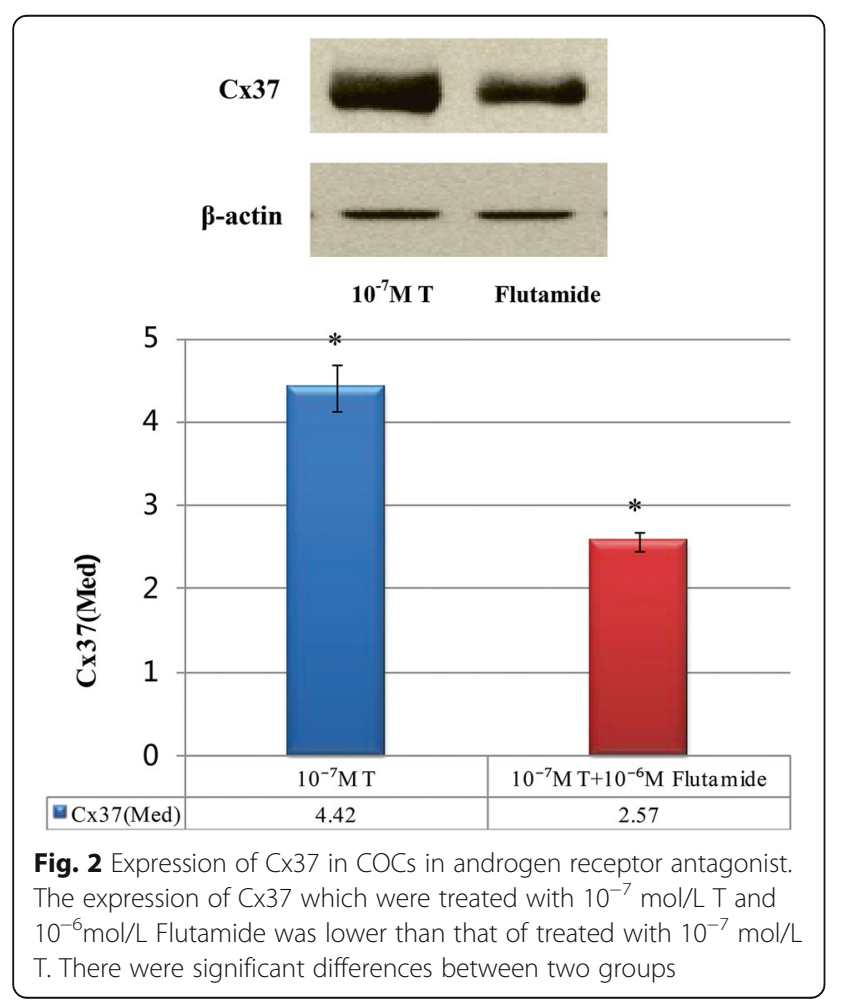

Recent researches report that there is a direct and indirect effect of androgen on follicle development. According to the two cell/two gonadotrophin theory, androgen plays an important role in ensuring adequate follicular steroidogenesis. Produced primarily by the theca cells, it acts as a substrate for the aromatase activity of the granlosa cells, and converts to estrogens (estradiol and estrone), sequentially plays a role in follicle growth [3]. This is called indirect regulation effect of androgen. Recent researches report androgen can also regulate follicle development directly through various mechanisms [4]. The mechanisms include the following several aspects: (1) androgen can increase one or more critical follicle growth factors concentration such as insulin-like growth factor-1 (IGF-1), growth differentiation factor-9 (GDF-9) in follicular fluid during the early stage of follicular development, which are known to have positive effect on follicular development and oocyte quality $[14,15]$; (2) androgen can augment granulosa cell follicle stimulating hormone receptor (FSHR) expression and improve follicle sensibility to FSH during the antral follicle stage [16]; (3) androgen via the AR pathway inhibits the expression of PTEN, and phosphorylate Akt proteins by phosphatidyl inositol 3kinase (PI3K)-Akt signaling pathways, thus induces primordium follicle activation [17]; (4) androgen can up-regulate the expression of $\mathrm{AR}$, promote granulosa cell proliferation and inhibit apoptosis [18].

Our previous studies have found that a significant decreased of connexin in cumulus cells is closely related to POR. In view of gap junctional intercellular communication is vitally important for follicular recruitment, growth and mature, and androgen plays an important role in improving prognosis of POR patients, we speculate that androgen can increase the expression of connexin in follicle cells, and improve ovarian microenvironment, thus promote ovarian response. To test this speculation, we treated COCs with testosterone of the equivalent of physiological concentrations in follicular fluid $\left(10^{-7} \mathrm{M}\right)$, low concentration $\left(10^{-9} \mathrm{M}, 10^{-11} \mathrm{M}\right)$, high concentration $\left(10^{-5} \mathrm{M}\right)$ and blank control group. The results suggested that compared to control group, testosterone-treated groups demonstrated an increasing amount of Cx37 protein in a dose-dependent manner. Combining with the preliminary study, our research suggested that adding a certain amount of androgen can improve the expression of connexin which is located the surface of oocytes and somatic cells in ovary, strengthen material and information communication among cells, improve ovarian micro-environment, thus improve ovarian response.

However, the mechanism of action of androgen on affecting the expression of connexin is unclear. AR appears on the surface of graunlosa cells when follicles 
start to improve, and the expression increases with follicle development. The expression of AR increases to peak on the surface of preantral follicle and small antral follicle, then begins to decline, and nearly cannot be detected on the surface of preovulatory follicle $[19,20]$. The variation of AR expression suggests that the function of androgen is different at the different stages of follicle development. Androgen can promote follicle recruitment and small antral follicle development through direct action in the early stage of follicle development; while most of androgen translates into estrogen and plays an indirect role in the follicle development, yet there is some direct effect. In this study, the expression of Cx37 in mouse COCs which were treated with androgen receptor antagonist was lower than the corresponding androgen group. The result suggested that androgen via the AR pathway played an important role in Cx37 expression. Further studies are required to understand the androgen-induced GJIC dependent signaling pathways mediating ovarian response.

\section{Conclusions}

This study suggested that androgen can improve the expression of connexin 37 which is located the surface of oocytes and somatic cells in ovary in a dose-dependent manner. There was close relationship between gap junction protein and ovarian response, which suggested that androgen could promote ovarian response by increasing the expression of Cx37 in follicle. Androgen via the AR pathway can promote the expression of connexin in COCs, strengthen material and information communication between oocytes and somatic cells, improve ovarian micro-environment, and thus improve ovarian response. The mechanism provides theoretical basis for adding androgen in treatment of POR in clinical. But the problem about rational use of time and dose of androgen still needs large sample clinical research.

\section{Abbreviations \\ AR: Androgen receptor; BCA: Bicinchoninic acid; COCs: Cumulus-oocyte-cells; DHEA: Dehydroepiandrosterone; DOR: Diminished ovarian reserve; ECL: Enhanced chemiluminescence; FSHR: Follicle stimulating hormone receptor; GDF-9: Growth differentiation factor-9; GJIC: Gap junction intercellular communication; hCG: Human chorionic gonadotrophin; HRP: Horseradish peroxidase; IGF-1: Insulin-like growth factor-1; IVF: In-vitro fertilization; LH: Luteinizing hormone; PBS: Phosphate-buffered saline; PCOS: Polycystic ovary syndrome; PMSG: Pregnant mare serum gonadotropin; POR: Poor ovarian response; SD: Standard deviation; SDS- PAGE: Sodium dodecyl sulfate polyacrylamide gel electrophoresisSPSS, Software Package for Social Sciences; TBS: Tris-buffered sal}

\section{Acknowledgements}

Not applicable.

Funding

Not applicable.

Availability of data and material Not applicable.

\section{Authors' contributions}

$Y Z$ and $Y X$ conceived and coordinated the study, designed and analyzed the experiments, wrote the paper. YK and SW performed the experiments. QX and IS carried out the data collection, data analysis, and revised the paper. All authors reviewed the results and approved the final version of the manuscript.

\section{Competing interests}

The authors declare that they have no competing interests.

\section{Consent for publication}

Not applicable.

\section{Ethics approval and consent to participate}

All procedures performed in studies involving animals were approved by the Animal Care and Use Committee of Peking University First Hospital. The animals used in this study were cared for, and used in accordance with ethical standards.

Received: 11 September 2016 Accepted: 10 November 2016 Published online: 23 November 2016

\section{References}

1. Kyrou D, Kolibianakis EM, Venetis CA, Papanikolaou EG, Bontis J, Tarlatzis BC. How to improve the probability of pregnancy in poor responders undergoing in vitro fertilization: a systematic review and meta-analysis. Fertil Steril. 2009;91(3):749-66. doi:10.1016/j.fertnstert.2007.12.077.

2. Bosdou JK, Venetis CA, Kolibianakis EM, Toulis KA, Goulis DG, Zepiridis L, et al. The use of androgens or androgen-modulating agents in poor responders undergoing in vitro fertilization: a systematic review and meta-analysis. Hum Reprod Update. 2012;18(2):127-45. doi:10.1093/humupd/dmr051.

3. Sunkara SK, Coomarasamy A, Arlt W, Bhattacharya S. Should androgen supplementation be used for poor ovarian response in IVF? Hum Reprod. 2012;27(3):637-40. doi:10.1093/humrep/der464.

4. Yang MY, Fortune JE. Testosterone stimulates the primary to secondary follicle transition in bovine follicles in vitro. Biol Reprod. 2006;75(6):924-32. doi:10.1095/biolreprod.106.051813.

5. Russell DL, Robker RL. Molecular mechanisms of ovulation: co-ordination through the cumulus complex. Hum Reprod Update. 2007;13(3):289-312. doi:10.1093/humupd/dml062.

6. Tong D, Gittens JE, Kidder GM, Bai D. Patch-clamp study reveals that the importance of connexin43-mediated gap junctional communication for ovarian folliculogenesis is strain specific in the mouse. Am J Physiol Cell Physiol. 2006;290(1):C290-7. doi:10.1152/ajpcell.00297.2005.

7. Wu CH, Yang JG, Yang JJ, Lin YM, Tsai HD, Lin CY, et al. Androgen excess down-regulates connexin43 in a human granulosa cell line. Fertil Steril. 2010;94(7):2938-41. doi:10.1016/j.fertnstert.2010.06.077.

8. Talhouk R, Tarraf C, Kobrossy L, Shaito A, Bazzi S, Bazzoun D, et al. Modulation of Cx43 and Gap Junctional Intercellular Communication by Androstenedione in Rat Polycystic Ovary and Granulosa Cells in vitro. J Reprod Infertil. 2012;13(1):21-32.

9. Lossl K, Andersen AN, Loft A, Freiesleben NL, Bangsboll S, Andersen CY. Androgen priming using aromatase inhibitor and hCG during earlyfollicular-phase $\mathrm{GnRH}$ antagonist down-regulation in modified antagonist protocols. Hum Reprod. 2006;21(10):2593-600. doi:10.1093/humrep/del221.

10. Casson PR, Lindsay MS, Pisarska MD, Carson SA, Buster JE. Dehydroepiandrosterone supplementation augments ovarian stimulation in poor responders: a case series. Hum Reprod. 2000;15(10):2129-32.

11. Wiser A, Gonen O, Ghetler Y, Shavit T, Berkovitz A, Shulman A. Addition of dehydroepiandrosterone (DHEA) for poor-responder patients before and during IVF treatment improves the pregnancy rate: a randomized prospective study. Hum Reprod. 2010;25(10):2496-500. doi:10.1093/humrep/ deq220.

12. Narkwichean A, Maalouf W, Campbell BK, Jayaprakasan K. Efficacy of dehydroepiandrosterone to improve ovarian response in women with diminished ovarian reserve: a meta-analysis. Reprod Biol Endocrinol. 2013;11: 44. doi:10.1186/1477-7827-11-44.

13. Weissman A, Horowitz E, Ravhon A, Golan A, Levran D. Dehydroepiandrosterone supplementation increases baseline follicular phase progesterone levels. Gynecol Endocrinol. 2011;27(12):1014-7. doi:10. 3109/09513590.2011.569611. 
14. Vendola K, Zhou J, Wang J, Bondy CA. Androgens promote insulin-like growth factor-l and insulin-like growth factor- receptor gene expression in the primate ovary. Hum Reprod. 1999;14(9):2328-32.

15. Hickey TE, Marrocco DL, Gilchrist RB, Norman RJ, Armstrong DT. Interactions between androgen and growth factors in granulosa cell subtypes of porcine antral follicles. Biol Reprod. 2004;71(1):45-52. doi:10.1095/biolreprod. 103.026484 .

16. Cardenas H, Herrick JR, Pope WF. Increased ovulation rate in gilts treated with dihydrotestosterone. Reproduction. 2002;123(4):527-33.

17. Yang JL, Zhang CP, Li L, Huang L, Ji SY, Lu CL, et al. Testosterone induces redistribution of forkhead box-3a and down-regulation of growth and differentiation factor 9 messenger ribonucleic acid expression at early stage of mouse folliculogenesis. Endocrinology. 2010;151(2):774-82. doi:10.1210/ en.2009-0751

18. Weil SJ, Vendola K, Zhou J, Adesanya OO, Wang J, Okafor J, et al. Androgen receptor gene expression in the primate ovary: cellular localization, regulation, and functional correlations. J Clin Endocrinol Metab. 1998;83(7): 2479-85. doi:10.1210/jcem.83.7.4917.

19. Juengel JL, Heath DA, Quirke LD, McNatty KP. Oestrogen receptor alpha and beta, androgen receptor and progesterone receptor mRNA and protein localisation within the developing ovary and in small growing follicles of sheep. Reproduction. 2006;131(1):81-92. doi:10.1530/rep.1.00704.

20. Rice S, Ojha K, Whitehead S, Mason H. Stage-specific expression of androgen receptor, follicle-stimulating hormone receptor, and anti-Mullerian hormone type II receptor in single, isolated, human preantral follicles: relevance to polycystic ovaries. J Clin Endocrinol Metab. 2007;92(3):1034-40. doi:10.1210/jc.2006-1697.

\section{Submit your next manuscript to BioMed Central and we will help you at every step:}

- We accept pre-submission inquiries

- Our selector tool helps you to find the most relevant journal

- We provide round the clock customer support

- Convenient online submission

- Thorough peer review

- Inclusion in PubMed and all major indexing services

- Maximum visibility for your research

Submit your manuscript at www.biomedcentral.com/submit

) Biomed Central 